\title{
Prospects for the use of a drug based on a complex of fatty acid esters in models of laboratory animals in vivo
}

\author{
K.Yu. Smirnova*, A.V. Bannikova, S.V. Kozlov, O.V. Romanova \\ Saratov State Agrarian University, 1, Teatralnaya square, Saratov, 410011, Russia
}

\begin{abstract}
In this work we carried out studies of the first obtained micellar composition of fatty acid esters with allantoin on a water basis to determine its local irritant effect and therapeutic efficacy in the treatment of burns. It was found that there was a sequential change in the phases of the burn wound process in all groups. During the observation period, wound suppuration was not observed; there were 4 cases of a complicated course of the wound process and a fatal outcome in the control group. When conducting studies of local irritant effects, the assessment was carried out in points, in the experimental group it was on average 2 points as for the animals of the control group. The conducted studies allow us to conclude that the drug, when applied to the skin according to the interstate standard GOST ISO 10993-10—2011, has a weak degree of response to irritation in guinea pigs. The application of a permissive dose of the drug to sensitized guinea pigs does not cause a response from the skin, which indicates the absence of allergenic properties in this composition.
\end{abstract}

\section{Introduction}

One of the important problems of veterinary medicine for many years has been the search for effective methods of treating infected wound processes in tissues [1]. This is due to the fact that the course of the wound process and the presence of anaerobic, aerobic microflora in the foci of infected inflammation lead to disruption of tissue respiration in the healing areas causing disturbances in the surrounding tissues, which ultimately result in the development of acidosis, hypoxia and metabolic intoxication of the body $[1,2]$.

Among the medicines for external use, ointments and gels are widely used with the bases that do not injure the damaged surface when applied to the wound thus providing a drainage of wounds. Medicinal substances that are part of such medicines provide the necessary therapeutic effect [2-5]. A promising direction is the development and application of multidirectional ointments that combine properties of antimicrobial activity, high dehydrating capacity, stimulation of tissue regeneration would be a source of investigation [6-10].

\section{Materials and methods}

We have developed a prototype of a wound-healing preparation based on fatty acid esters. This sample was obtained by pressing the biomass of Hermetia illucens larvae. When developing a prototype of the drug, we obtained 34 samples. The most optimal was Sample 6 that consists a complex of fatty acid esters - $13 \%$, allantoin - $10 \%$, benzyl alcohol - $1 \%$, dimethyl sulfoxide (DMSO) - 10\%, carboxylmethylcellulose (CMC) $-1 \%$, glycerin - 5\%, talc - $33 \%$, aloe juice - 10\%, TWIN-80 $5 \%$ and water.

To prepare the animals for testing for the local irritant effect of the drug, the hair on the experimental skin areas was carefully cut before the start of the study.

The dose administered intradermally to each study area was $0.1 \mathrm{ml}$.

For applications, the test sample was impregnated with filter paper or a hygroscopic gauze pad (4-8 cm2) which was applied to the clipped skin areas and fixed with an occlusive dressing around the animal's body.

Preliminary tests were carried out in order to determine the concentration of the test sample to be used in the main study.

Undiluted extracts using known solvents do not need to be tested beforehand.

Consideration should be given to the possible effect of the administration of complete Freund's adjuvant (FCA) in combination with the test material during the main test and thus the possible bias of the results.

According to the application, extreme dilutions were tested on three animals.

After 24 hours, the dressing and samples were removed and the condition of the test sites was assessed for the presence of erythema and edema in accordance with Table 1 (according to the classification of Magnusson and Kligman).

For the induction phase in the main test, the highest concentration was chosen which does not cause more than very weak erythema, and does not have an overall adverse effect on the animals.

\footnotetext{
*Corresponding author: smirnova.ku@mail.ru
} 
Table 1. Skin reaction classification system

\begin{tabular}{|c|c|}
\hline $\begin{array}{c}\text { Description of the } \\
\text { response }\end{array}$ & Points \\
\hline No visible changes & 0 \\
\hline $\begin{array}{c}\text { Discrete or focal } \\
\text { erythema }\end{array}$ & 1 \\
\hline $\begin{array}{c}\text { Moderate to } \\
\text { continuous erythema }\end{array}$ & 2 \\
\hline $\begin{array}{c}\text { Intense erythema and } \\
\text { swelling }\end{array}$ & 3 \\
\hline
\end{tabular}

\subsection{Intradermal induction phase}

Each animal received paired intradermal injections in a volume of $0.1 \mathrm{ml}$ into the clipped skin areas (A, B and C) in accordance with Figure 1.

$$
1
$$

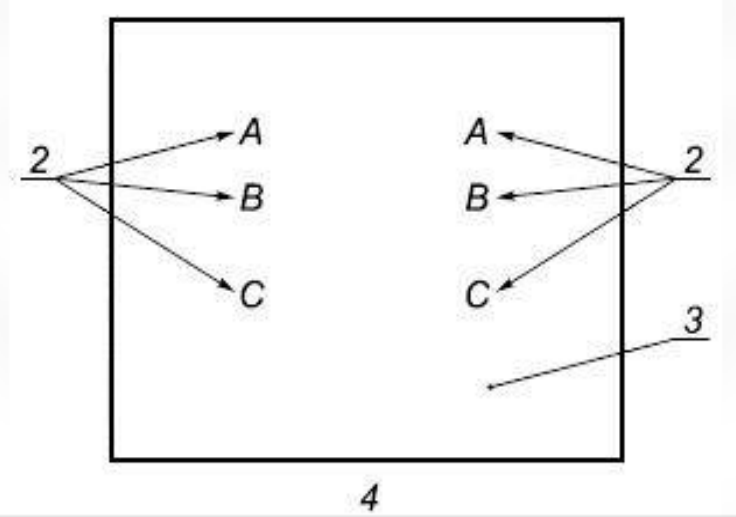

Fig. 1. Location of intradermal injection sites; 1 - head; 2 - 0.1 $\mathrm{ml}$ intradermal injection; 3 - clipped intra-scapular region; 4 tail

A - Mixture of complete Freund's adjuvant with the selected solvent in a 50/50 ratio. We used physiological saline solution (according to British Pharmacopoeia, USP or equivalent) for water-soluble materials.

B - Test sample (undiluted extract); control animals were injected only with the vehicle.

C - Test sample at the concentration selected for the site, emulsified with complete Freund's adjuvant in a $50 / 50$ ratio and solvent $(50 \%)$; control animals received only the emulsion of the control solution with adjuvant.

\subsection{Local induction phase}

Seven days ( \pm 1 day) after the intradermal induction phase dermal applications of the test material were started on the injection sites, on the intra-scapular region of each animal using soaked pieces of gauze with an area of $8 \mathrm{~cm}^{2}$.

In this case, the maximum concentration for site $\mathrm{B}$ was used. If the maximum concentration that can be achieved does not cause irritation, the area of application was pretreated with a $10 \%$ solution of sodium dodecyl sulfate, massaged into the skin $24 \pm 2$ hours before application. An occlusive dressing was applied. The bandage was removed after $(48 \pm 2) \mathrm{h}$.
Freshly prepared extracts were used. If the extracts were prepared earlier than $24 \mathrm{~h}$, then the stability of the extracts during storage was checked.

The procedure with control animals was repeated in the same mode using only the control solution.

\subsection{Provocative phase}

14 days ( \pm 1 day) after the end of the local induction phase, all experimental and control animals were challenged with the test material. For this, the test material was applied to the intact skin areas of the sides of each animal using a hygroscopic gauze pad. A fixing occlusive dressing was applied. The bandage and applications were removed after $(24 \pm 2) \mathrm{h}$.

\subsection{Inspection of animals}

Examined the surface of the areas where the provocative test was carried out in experimental and control animals, after 24, 48 hours after removing the bandage. Full spectrum illumination was used to visualize skin reactions.

Described and assessed the degree of skin reaction, including erythema and edema in accordance with Table 1 (according to the classification of Magnusson and Kligman) for each site and at each time interval of observation.

The description was made without taking into account the preliminary impact in order to minimize the error in assessing the results.

Laboratory animals - rats - served as the material for research on the therapeutic efficacy in burns. Work was carried out on the formation of experimental and control groups of animals on the principle of analogs. At the time of the study, the animals (rats) were clinically healthy.

The studies were carried out in accordance with the "Rules of laboratory practice in the Russian Federation" (Order of the Ministry of Health of the Russian Federation No. 708n dated 23.08.2010). Experiments on animals were carried out in accordance with the rules adopted by the European Convention for the Protection of Vertebrate Animals Used for Experimental and other Scientific Purposes (ETS 123) Strasbourg, 1986).

The studies were performed according to an approved written protocol and in accordance with the Investigator's Standard Operating Procedures (SOP).

The study subjects were rats that were identified and evaluated for the study variable on an individual basis.

All animals were examined, which included a clinical study.

Based on the data obtained in the course of the above studies, groups were formed according to similar physiological parameters.

The study included 30 clinically healthy rats. The experimental animals were kept under the same conditions, optimal for the given species. The animals were kept in cages for 10 heads. Before the start of the experiment, all cells were labeled. 
The pharmacological activity of the ointment - antiburn and wound healing - was determined on a model of thermal burn in rats.

The day before the burn was applied, the hair on the back area of $10 \mathrm{~cm}^{2}$ was removed from the experimental animals. On the day of the experiment, rats were anesthetized with isoflurane.

After the animals were injected into anesthesia, they were placed on a table for fixing laboratory animals. Experimental burn wounds were modeled using a metal rod with a surface diameter of $1 \mathrm{~cm}^{2}$, which was heated to $100{ }^{\circ} \mathrm{C}$. The rod was applied to the animal's skin for $20 \mathrm{sec}$. As a result, a IIIA degree burn with a total area of $100-130 \mathrm{~mm}^{2}$ was obtained.

After inclusion in the study, the animals were assigned to one of three groups (Table 2):

- 1 experimental group $(n=10)$ - an experimental sample of the cream "Sample No. 6" was applied to the burn wound to the animals for 20 days;

- 2 experimental group $(n=10)$ - Panthenol universal cream was applied to the burn wound to animals for 20 days;

- the third group $(n=10)$ of animals was control, the burn wound remained untreated.

Experimental groups of animals received gels at a dose of $0.5 \mathrm{~g}$ immediately after the application of the burn and then daily.

Every day from the beginning of the experiment, the general condition of the rats was recorded.

The area of experimental burns was measured by a planimetric method using a caliper. The diameter of the burn was measured, then its area was calculated using the formula: $S=2 \pi R 2$.

Measurement of the area of the wound was performed on the $1 \mathrm{st}, 5 \mathrm{th}, 10 \mathrm{th}$ and 20 th days after the application of the burn wound.

The general condition of the animals was monitored on the basis of behavioral responses.

Table 2. Experiment design

\begin{tabular}{|c|c|l|c|}
\hline Group & Drug/scheme & $\begin{array}{l}\text { Method of } \\
\text { application }\end{array}$ & $\begin{array}{l}\text { Number } \\
\text { of } \\
\text { animals }\end{array}$ \\
\hline $\begin{array}{c}\text { 1st } \\
\text { experimental }\end{array}$ & $\begin{array}{c}\text { Experimental } \\
\text { cream sample } \\
\text { "Sample No. 6" }\end{array}$ & Outwardly & 10 \\
\hline $\begin{array}{c}\text { 2nd } \\
\text { experimental }\end{array}$ & $\begin{array}{c}\text { Panthenol } \\
\text { Cream }\end{array}$ & Outwardly & 10 \\
\hline 3rd control & \multicolumn{2}{|c|}{-10} \\
\hline
\end{tabular}

\section{Results and discussion}

When studying the local irritant effect after paired intradermal injection of substances in the corresponding areas of the skin in a volume of $0.1 \mathrm{ml}$, each animal was recorded after 24 hours.

In the control group, there was a slight swelling at the injection site which disappeared after 6-8 hours; the condition of the animals was stable, erythema was not observed. In the experimental group of animals, at the injection site of a mixture of Freund's complete adjuvant with the selected solvent in a 50/50 ratio there was a slight swelling at the injection site which disappeared after 6-8 hours; the condition of the animals was stable, and a slight erythema was observed. At the injection site of the test sample with a solvent, there was a slight swelling at the injection site which passed after 4-5 hours; the condition of the animals was slightly depressed, but after 1-2 hours it was stable, erythema was not observed.

After the introduction of the test sample at the concentration selected for the site emulsified with complete Freund's adjuvant in a 50/50 ratio and a solvent (50\%), a slight swelling was observed at the injection site which passed after 6-8 hours, the condition of the animals was slightly depressed, but after $2-3$ hours stable, no erythema was observed.

Seven days ( \pm 1 day) after the intradermal induction phase, dermal applications of the test material were started on the injection sites, on the intra-scapular region of each animal using soaked pieces of gauze with an area of $8 \mathrm{~cm}^{2}$. The surface of the skin areas of animals within 14 days after the application of skin applications of the test material in experimental and control animals was examined.

At 24 hours after removing the dressing, moderate erythema was observed in all animals, including controls. After 48 hours, a small discrete or focal erythema was observed in the first and second experimental groups of animals. After 7 days, there was a slight swelling together with the introduction in all groups of animals. After 14 days no changes were observed in all animals of the control and experimental groups. The score in points obtained in the experimental group is on average 2 points, as in the animals of the control group.

In this regard, the studies conducted allow us to conclude that the drug, when applied to the skin according to the interstate standard GOST ISO 1099310-2011 has a weak degree of response to irritation in guinea pigs.

The application of a permissive dose of the drug to sensitized guinea pigs does not cause a response from the skin that indicates the absence of allergenic properties in this composition.

In the course of the study to determine the therapeutic efficacy for burns, it was found that in all groups there was a sequential change in the phases of the burn wound process. During the observation period, wound suppuration was not observed; there were 4 cases of death in the control group and a complicated course of the wound process.

On the second day after the application of the burn injury, skin hyperemia along the periphery of the burn was noted in all groups of animals. In the area of the burn, necrosis is noted, in some places only the surface layer of the skin itself is affected, in others the burn extends to its entire thickness, accompanied by complete necrosis of the papillary layer. A superficial dry whitishgray scab is formed. Outwardly, there is no difference between animals of different groups.

By the seventh day of the experiment, the appearance of pink-red skin papillae over the entire surface of the wound was noted in the first experimental group of 
animals. The formation of granulation tissue and the beginning of epithelialization from the side of healthy skin occurs. The area of the wound is reduced (Figure 2).

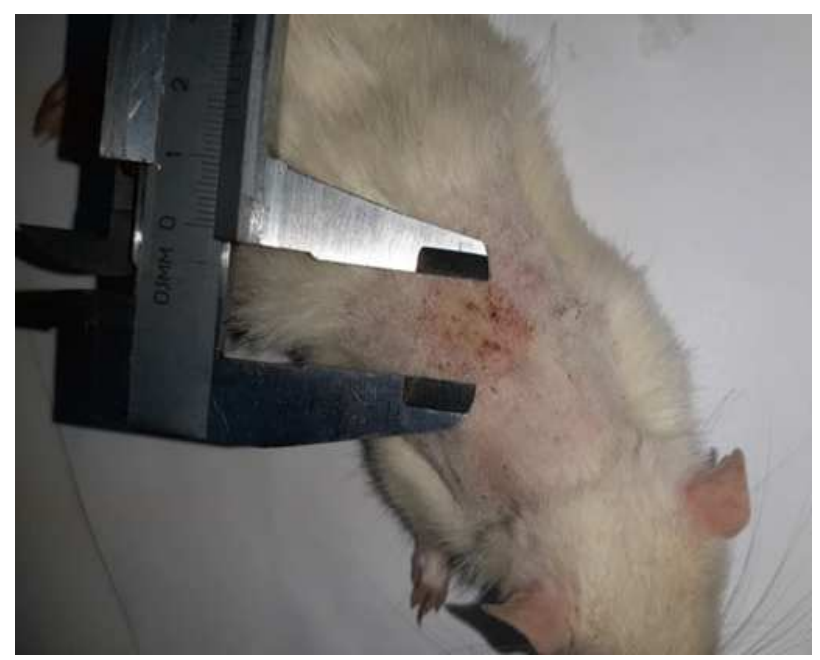

Fig. 2. The result on the 7 th day after the experimental application of burns in the 1st experimental group (Sample No. 6)

In animals of the second experimental group, which received the commercial preparation Panthenol as an anti-burn agent, the regenerative processes are more pronounced. On the periphery of the wound, there is intense epithelialization from the side of healthy skin. The wound is noticeably reduced in size. Most of the burn wound is covered with a scab, which begins to be rejected by overgrown epithelial cells (Figure 3).

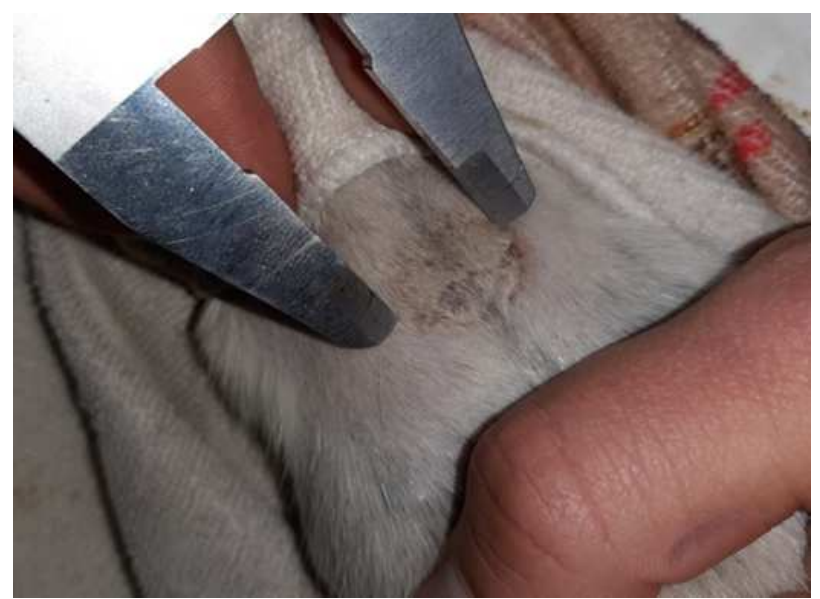

Fig. 3. The result on the 7 th day after the experimental application of burns in the 2nd experimental group (Panthenol)

At the same time, in the control group of animals, by this time, the formation of a demarcation shaft between healthy and necrotic tissues was observed. The surface of the burn has a variegated appearance. Against the background of whitish-gray necrotic tissues, pink-red skin papillae are just beginning to appear (Figure 4).

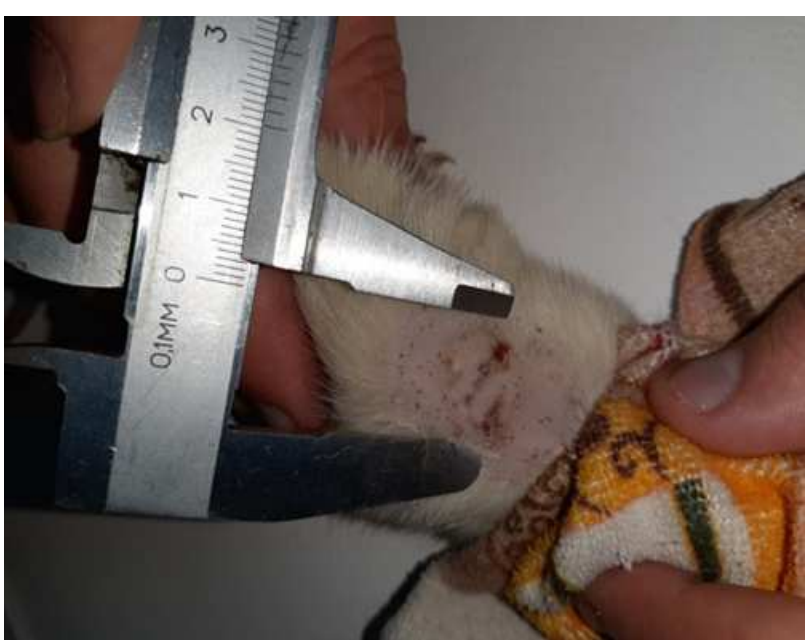

Fig. 4. The result on the 7 th day after the experimental application of burns in the control group

On the 14th day of the experiment, in the animals of the first experimental group, which used "Sample No. 6" as an anti-burn agent, there was a pronounced positive dynamics. The wound noticeably decreases in volume, islands of epithelialization are visible on granulations, a noticeable rejection of the scab occurs (Figure 5).

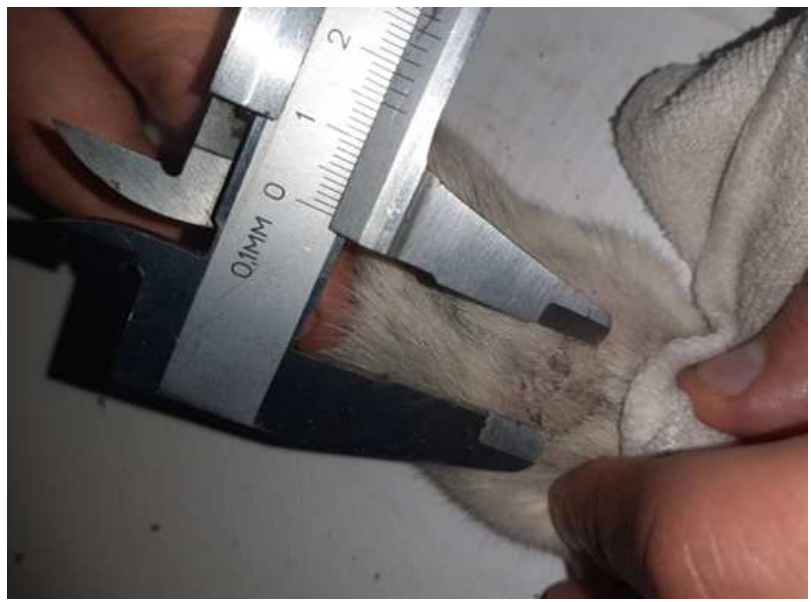

Fig. 5. The result on the 14 th day after the experimental application of burns in the 1 experimental group (Sample No. 6)

At the same time, in the animals of the second experimental group, which were prescribed the commercial drug Panthenol as an anti-burn agent, an intensive positive dynamics of reparative processes was also observed. The volume of the wound is reduced 2 times. Intensive epithelialization occurs on the part of healthy skin (Figure 6).

In the control group of animals, on the 14th day after the application of the burn injury, weak positive changes in the wound were noted. The condition in 4 animals was worsened, lethargy, hypersalivation, a decrease in body temperature and depression were noted. The repair occurs very slowly and exclusively due to the physiological mechanisms of the body. There is an alteration, severe hyperemia, autolysis of cells, the disintegration of their connective tissue under the action of collagenase, ellastase, destroying the protein 
backbone of the connective tissue. On the 17th day, the death of 4 animals of the control group is observed.

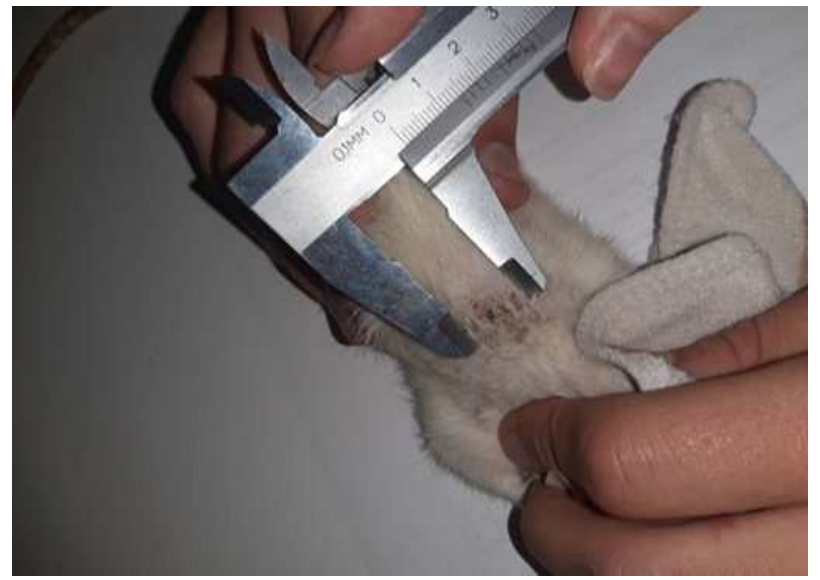

Fig. 6 - The result on the 14th day after the experimental application of burns in the 2nd experimental group (Panthenol)

By the 20th day of the experiment, in the first experimental group of rats, complete healing of the wound surface is noted. In the second experimental group, on the 20th day of the experiment, complete healing of the wound surface is also noted. In the control group of animals, by the 20th day of the experiment, physiological processes of relief of the burn wound were noted. Intense epithelialization on the part of healthy skin. A noticeable decrease in the volume of the wound surface. 4 animals were fatal.

\section{Conclusions}

Currently, thermal burns account for one tenth of the total number of all injuries in the world [11]. It has been established that improper and untimely treatment of skin burns entails negative consequences for the body: infection and deepening of the wound, inhibition of the regeneration process, and the formation of excessive scarring [12]. For effective therapy, it is necessary to use multicomponent drugs with antiseptic and wound healing effects.

When conducting studies to determine the local irritating effect of the drug based on fatty acid esters after applying cutaneous applications after 7 days, there was a slight swelling together with the introduction in all groups of animals. After 14 days, no changes were observed in all animals of the control and experimental groups.

During the observation period, no suppuration of wounds was observed, 4 cases of death in the control group and a complicated course of the wound process were noted. On the second day after the application of the burn injury, there was no externally observed difference between the animals of different groups. By the seventh day of the experiment, in the first experimental group of animals, the formation of granulation tissue and the beginning of epithelialization from the side of healthy skin take place. On the 14th day of the experiment, in the animals of the first experimental group, islands of epithelialization are visible on granulations, there is a noticeable rejection of the scab. By the 20th day of the experiment, in the first experimental group of rats, there is a complete restoration of epithelial tissue, growth of hair on the side of healthy skin.

Thus, on the basis of the above, we can conclude that the obtained experimental sample of the preparation "Sample No. 6" has pronounced wound-healing properties. This is evidenced by the reduction in the time of complete restoration of the skin after the application of a burn injury to rats in comparison with control animals. The prototype cream "Sample No. 6" has a wound-healing effect on a par with the conventional means "Panthenol", and also has a low local irritant effect as evidenced by the results of intradermal injection of the sample and the imposition of skin applications

\section{References}

1. N.A. Barsukov, Infectious wounds healing, Veterinary, 8, 68-69 (1986)

2. J.S. Boateng, K.H. Matthews, H.N. Stevens, G.M. Eccleston, Wound healing dressings and drug delivery systems: a review, J. Pharm. Sci., 97, 2892923 (2008)

3. I.V. Alekseyeva, Development of pharmaceutical forms for wounds healing, Pharmacy, 2, 43-45 (2003)

4. C.C. Bulik, D.E. Wiskirchen, A. Shepard, et al., Tissue Penetration and Pharmacokinetics of Tigecycline in Diabetic Patients with Chronic Wound Infections Described by Using In vivo Microdialysis, Antimicrob. Agents Chemother., 54, 5209-5213 (2010)

5. A. Francesko, Chitin, chitosan and derivatives for wound healing and tissue engineering, Advances in biochemical engineering/biotechnology, 1-27 (2011)

6. N.A. Kuznetsov, V.G. Nikitin, Gentle surgical interventions and interactive dressings in the treatment of infected wounds, Consilium medicum: Surgery (app.), 2, 39-46 (2006)

7. A. Karami, H. Tebyanian, A. Barkhordari, E. Motavallian, R.S. Soufdoost, M.R. Nourani, Healing effects of ointment drug on full-thickness wound, C. R. Acad. Bulg. Sci., 72, 123-129 (2019)

8. L.A. Blatun, Local medicated wounds healing. Problems and new possibilities of its solution, Consilium medicum: surgery (supplement), 1, 9-16 (2007)

9. O.S. Larionova, YA.B. Drevko, A.V. Bannikova, A.S. Kovtunova, D.V. Mendubayev, Yu.S. Karmeyeva, L.S. Krylova, Method for the production of chitosan. Patent RU 2615636 C, 6 April 2017. Application No. 2016110254, 21 March 2016.

10. A.V. Volenko, A.L. Men'shikov, G.P. Titova, S.V. Kuprikov, Prevention of wound infection with immobilized antibacterial drugs, Surgery, 10, 54-58 (2004) 
11. F. Yang, et al., Pathogenic alteration in severe burn wounds, J. Burns., 38(I.1), 90-94 (2012)

12. L.S. Krylova, Ye.K. Remizov, K.Yu. Smirnova, O.S. Larionova, Indication of peptides from the insect larva biomass and study of their antimicrobial activity, Current issues of veterinary biology, 4(44), 3-6 (2019) 